Weitere Themen sind für die nächsten Informationstage vorgesehen:

- Das Konzept von DAR und TGA;

- Qualitätssicherung im analytischen Laboratorium;

- Struktur und Aufgaben einer Akkreditierungsstelle.

Die europaweite Förderung der Qualität analytischer Resultate ist die wichtigste Leitlinie von EURACHEM. Diesem Ziel dienen Workshops, die sich teils in Vorbereitung befinden, teils schon in einer ersten Version stattgefunden haben ("Quality Assurance" im September 1991 in Straßburg). Sie sollen als Vorgabe dienen für entsprechende Kurse für Analytiker in den Mitgliedsländern. EURACHEM/D wird in 1993 die Planung derartiger Veranstaltungen im Rahmen des GDCh-Fortbildungsprogramms aufnehmen, die folgende Themen umfassen sollen:

- Qualitätssicherung im analytischen Laboratorium;

- Durchführung und Interpretation von Ringversuchen;

- Vergleichbarkeit und Rückführbarkeit von Meßwerten auf internationale Standards;

- Einsatz und Nutzen von Referenzmaterialien.

Zur Harmonisierung der nationalen Akkreditierungssysteme wird die Anwendung der Normenserie EN 45000 hilfreich sein. Die europaweite Harmonisierung hoher Qualität und Zuverlässigkeit analytischer Ergebnisse ist damit aber noch nicht zwangsläufig verbunden. EURACHEM versucht, durch Förderung des Qualitätsgedankens in der Analytik und durch Verbreitung der dafür erforderlichen Kenntnisse die Voraussetzungen zu schaffen, daß die Akkreditierung nicht zur lästigen Formalie wird, sondern daß deren angestrebtes Ziel auch wirklich erreicht werden kann.

Der Arbeitskreis EURACHEM/D ist bezüglich der Mitgliedschaft offen für alle von Akkreditierungs- und Zertifizierungsfragen betroffenen Personen, Unternehmen, Laboratorien, Organisationen und Gremien aus der Wirtschaft und aus dem staatlichen Bereich. Die Mitgliedschaft in der GDCh ist nicht Voraussetzung. Zur Deckung seiner Kosten (Teilnahme an den internationalen Sitzungen von EURACHEM und dessen Arbeitsgremien, Mitgliedschaften in relevanten nationalen und internationalen Organisationen, Drucksachen, Porto usw.) erhebt der Arbeitskreis einen Jahresbeitrag von DM $500,-$.

Interessenten an einer Mitgliedschaft im Arbeitskreis EURA$\mathrm{CHEM} / \mathrm{D}$ wenden sich bitte an die

Gesellschaft Deutscher Chemiker Sekretariat EURACHEM/D Postfach 900440 D-6000 Frankfurt (Main) 90

\title{
Fachgruppe Umweltchemie und Ökotoxikologie der GDCh
}

\section{- Jahrestagung, 16. - 17. November 1992 in Potsdam}

\section{Leitung: Prof. Dr. O. HutzINGER, Vorsitzender der Fachgruppe Umweltchemie und Ökotoxikologie.}

Vertreter der Umweltbehörde der NRW-Landeshauptstadt Düsseldorf, des Umweltbundesamtes und der C.A.U. Frankfurt/Main in referierten im Themenbereich Stoffkreisläufe über die Aspekte: "Ökobilanzen für Produkte“, „Nachwachsende Rohstoffe“, „Kunststoff-Folien".

Ergebnis:

1. Die notwendigen Instrumente zur Steuerung von Stoffströmen wie Stoffstrombilanzen, Produktlinienanalysen/Ökobilanzen und ökonomische Hebel sowie freiwillige Vereinbarungen müssen zu einem umwelt- und wirtschaftspolitischen Ganzen zusammengefügt werden.

2. Recycling ist nur die zweitbeste Lösung, wie Untersuchungsberichte dieser Veranstaltung zeigten.

Behandelt wurden

- der Rahmen eines Ökobilanz-Standardmodells mit den Untersuchungsschritten Vertikalanalyse, Horizontalanalyse, Wirkungsbilanz und Bilanzbewertung,

- die Berücksichtigung zusätzlicher Kriterien (Flächenbedarf, Pflanzenschutz- und Düngemittel sowie deren Emissionen) bei Ökobilanzen Nachwachsender Rohstoffe,

- die Eignung von Ökobilanzen zur ökologischen Beurteilung von Packmitteln und Verpackungssystemen.
Wissenschaftler der Universitäten Tübingen, Marburg, Bonn, Wuppertal, Bochum, der C.A.U., von Landesumweltämtern sowie aus Instituten und wissenschaftlichen Einrichtungen in Potsdam und Hamburg nahmen weiterhin Stellung zu biochemischen Indikatoren der Schadstoffbelastung.

Der abiotische Abbau mittelflüchtiger organischer Verbindungen (SOC) in der Troposphäre wurde abgeschätzt und hinterfragt.

Im Zusammenhang damit wurden Fortschritte und Grenzen gegenwärtiger Prüfmethoden der $\mathrm{OH}$-Reaktivität behandelt und die Wiederherstellung von Bodenfunktionen durch extensive Landwirtschaft am Beispiel ausgewählter Niedermoorflächen Brandenburgs erläutert.

In Postern zu Umweltbelastungen, Belastungsquellen, Recycling, Abbau, Abfall, Analytik und Nährstoffeintrag in die Umwelt wurden wichtige Informationen zur Schadstoffaufklärung gegeben. Weitere Themen der Poster waren Ökotoxikologie, Toxikologie, die atmosphärische Bleibelastung in der Industrieregion Leipzig-HalleBitterfeld, der N-Stoffwechsel der Kiefernnadeln als Bioindikationssystem von Umweltkontaminationen, neuronale Netze zur Untersuchung von Struktur-Wirkungs-Beziehungen sowie das Depositionsmeßnetz von Bayern zum Vergleich zwischen Bulk- und Wet-onlyNiederschlagssammlung.

Die Redaktion

\section{Gründungsjubiläum der Deutschen Chemischen Gesellschaft}

Vor 125 Jahren, am 11. November 1867, wurde in Berlin die Deutsche Chemische Gesellschaft gegründet, aus der nach dem Zweiten Weltkrieg die heutige Gesellschaft Deutscher Chemiker (GDCh) hervorging. Einer ihrer Gründer und ihr erster Präsident war der Chemiker August Wilhelm von HoFMANN, dessen Arbeiten über das Anilin und seine Abkömmlinge den Weg für die Entwicklung der modernen Farbenchemie ebneten. Anläßlich dieses Jubiläums verlieh die GDCh am 11. November 1992 Auszeichnungen an zwei bedeutende Chemiker: Die August-Wilhelm-von-HofmannDenkmünze wurde an Professor Sir Alan BATTERSBY, University of Cambridge, in Anerkennung seiner Forschungsarbeiten über die Biosynthese von physiologisch wirksamen Substanzen verliehen. Die zweite Auszeichnung fand in Form der August-Wilhelmvon-Hofmann-Vorlesung (Gedächtnis-Vorlesung) statt und wurde in diesem Jahr von Professor Dr. Richard ERNST (Nobelpreisträger 1991) gehalten. 